\title{
Retraction
}

\section{Retracted: Gabor Weber Local Descriptor for Bovine Iris Recognition}

\section{Mathematical Problems in Engineering}

Received 12 November 2013; Accepted 12 November 2013

Copyright (C) 2013 Mathematical Problems in Engineering. This is an open access article distributed under the Creative Commons Attribution License, which permits unrestricted use, distribution, and reproduction in any medium, provided the original work is properly cited.

The paper titled "Gabor Weber Local Descriptor for Bovine Iris Recognition" [1], published in Mathematical Problems in Engineering, has been retracted upon the authors' request as it was found to include erroneous data. Its findings and conclusion cannot be relied on.

\section{References}

[1] S. Sun, L. Zhao, and S. Yang, "Gabor Weber local descriptor for bovine iris recognition," Mathematical Problems in Engineering, vol. 2013, Article ID 920597, 7 pages, 2013. 


\title{
Gabor Weber Local Descriptor for Bovine Iris Recognition
}

\author{
Shengnan Sun, Lindu Zhao, and Shicai Yang
}

Institute of Systems Engineering, Southeast University, Sipailou 2, Nanjing 211189, China

Correspondence should be addressed to Lindu Zhao; ldzhao@seu.edu.cn

Received 27 February 2013; Revised 12 April 2013; Accepted 29 April 2013

Academic Editor: Rongni Yang

Copyright ( 2013 Shengnan Sun et al. This is an open access article distributed under the Creative Commons Attribution License, which permits unrestricted use, distribution, and reproduction in any medium, provided the original work is properly cited.

Iris recognition is a robust biometric technology. This paper proposes a novel local descriptor for bovine iris recognition, named Gabor Weber local descriptor (GWLD). We first compute the Gabor magnitude maps for the input bovine iris image, and then calculate the differential excitation and orientation for each pixel over each Gabor magnitude map. After that, we use these differential excitations and orientations to construct the GWLD histogram representation. Finally, histogram intersection is adopted to measure the similarity between different GWLD histograms. The experimental results on the SEU bovine iris database verify the representation power of our proposed local descriptor.

\section{Introduction}

The food safety and security has become one of the hot topics noticed widely by the society in recent years due to its harm to the health. Traceability system is considered to be an effective solution to meet heightened consumer expectations. Identification technology is crucial for the traceability system of meat supply chain. In existing traceability systems, different identification technologies have been adopted, such as ear tag [1], tattoo, and RFID [2]. Although these technologies have achieved some results, they still have deficiency to support the traceability system, as these technologies rely on manual code. Recently, a tracking and traceability system for meat supply chain based on iris recognition was proposed by Zhao et al. [3] to ensure food safety.

Iris recognition has gained popularity as a robust and reliable biometric technology for its characteristics of uniqueness, stability, and noninvasiveness of iris [4]. The iris's complex texture and its apparent stability hold tremendous promise for leveraging iris recognition in diverse application scenarios, such as border control, forensic investigations, and cryptosystems [5]. One of the key procedures for iris recognition systems is feature representation. Feature representation aims to describe the unique iris texture pattern, which should be highly distinctive among iris images of different eyes while maintaining invariance among iris images of the same eyes [6].
The most common practice is to generate the feature representation by extracting the spatial frequency information from the iris texture with different filters. The wellstudied filter is 2D Gabor filter, proposed by Daugman [4], as the Gabor filter has shown to be optimal in the sense of minimizing the joint two-dimensional uncertainty in space and frequency. Some other researchers also considered the application of Gabor filter, its variant, or other filters to extract texture information. Considering the ordinal Gabor filter would under represent the high frequency components in natural images, Yao et al. [7] used modified Log-Gabor filters to generate iris code. Wildes [8] applied Laplacian of Gaussian filters to encode the iris texture. Ma et al. [9] used a variant of the Gabor filter at two scales to analyze the iris texture, and generated the feature vector with Fisher's linear discriminant. Sun et al. [10] used a Gaussian filter to compute the local orientation at each pixel in the unwrapped template and then used the binary code quantized from the angle to represent iris image. Monro et al. [11] applied discrete cosine transform (DCT) to generate a binary code of iris texture by calculating the differences between the DCT coefficients. Miyazawa et al. [12] proposed to use Fourier transform to compute the representation of iris texture.

In the past years, local descriptors, which represent the image using local characteristic directly in the spatial domain, have been proven to be successfully in image retrieval, image classification, and object recognition. Several popular local 
descriptors have been introduced to iris recognition, such as local binary pattern (LBP) [13], scale invariant feature transform (SIFT) [14], and DAISY [15]. Unlike the spatial frequency methods, local descriptors extract image properties directly in the spatial domain. Sun et al. [16] proposed an efficient graph-matching scheme for iris recognition, by using LBP to generate the histogram representation of iris texture. Belcher and Du [17] applied the SIFT to the iris recognition. They detected keypoints in the iris images, and represented these keypoints using descriptor of orientation histogram. Zhang et al. [18] designed the Deformable DAISY Matcher (DDM) for iris recognition, based on the DAISY descriptor.

Recently, several literatures suggested to combine local descriptor with Gabor filter to improve its performance, as the Gabor filter has shown its high discriminant ability. Zhang et al. [19] proposed the so-called local Gabor binary pattern histogram sequence (LGBPHS) for face representation, by combining Gabor filter with LBP, and Takahashi et al. [20] applied spatiotemporal Gabor filtering to bicycle detection together with Histogram of Oriented Gradients (HOG) [21].

While the texture of bovine iris images is not as salient as human iris images and there exist noise and illumination in the bovine iris images, it is difficult for traditional feature representation algorithms to achieve high performance, which further affects the application of bovine iris recognition technology in food safety. Therefore, a more accurate bovine iris representation is required. Motivated by Weber local descriptor (WLD) [22] and the combination of Gabor filter and local descriptor [20,21], we propose a novel local descriptor for bovine iris recognition, named Gabor Weber local descriptor (GWLD). The novelty of the GWLD lies in generating the histogram representation by computing the differential excitation and gradient orientation over these Gabor magnitude maps of the bovine iris image. The combination of Gabor and WLD enhances the representation power of the spatial histogram for bovine iris recognition greatly.

The remaining part of the paper is organized as follows. Section 2 introduces the preprocessing of bovine iris images. Section 3 describes the computation scheme of GWLD and bovine iris matching algorithm. And experimental results are shown in Section 4. At last, the conclusion is given in Section 5.

\section{Bovine Iris Image Preprocessing}

Similar to human iris recognition, bovine iris recognition usually involves several crucial steps: image acquisition, image preprocessing, feature extraction, and iris matching. Image preprocessing includes iris localization, iris normalization, and enhancement. In this paper, we use the image preprocessing procedure mentioned in our previous work [23]. The preprocessing method is briefly described as follows. Firstly, the inner and outer iris edge curves are detected by region-based active contour model, which uses the global and local intensity information to guide the contour evolution. Secondly, the inner and outer iris edge curves are used to fit ellipses with least square method, and then elliptic iris boundaries are obtained. Thirdly, to eliminate the influence of eyelid and lash, which usually exists in the original bovine iris image, $[-\pi / 4, \pi / 4]$ and $[3 \pi / 4,5 \pi / 4]$ are selected to generate the normalized rectangle iris block with polar coordinate transformation. Finally, the normalized bovine iris block is enhanced with histogram equalization.

Figure 1(a) shows the original bovine iris image with initial contour, Figure 1(b) illustrates the final evolution curves after 50 iterations by using the region-based active contour model, and Figures 1(c), 1(d), and 1(e) show the elliptic iris boundaries, normalized bovine iris image, and enhanced iris block, respectively.

\section{Bovine Iris Representation and Matching}

We first describe how to compute the Gabor Weber local descriptor in Section 3.1 and then introduce the bovine irismatching algorithm using GWLD.

3.1. Computation of GWLD. Weber local descriptor [22], inspired by the Weber's Law, was proposed as a simple and powerful image descriptor and considered to be able to combine the advantages of LBP [13] in computing the intensity difference pixel-wise and SIFT [14] in using gradient information. The WLD describes the intensity relationship between a pixel and its neighborhoods with two components: differential excitation and orientation.

The overall framework of the proposed bovine iris representation approach based on GWLD is illustrated in Figure 2. In this approach, the bovine iris image is modeled as a GWL histogram by the following procedures: (a) an input bovine iris image is transformed to obtain multiple Gabor magnitude maps in frequency domain by applying multiscale and multiorientation Gabor filters; (b) compute the differential excitation and orientation for each pixel over each Gabor magnitude map; (c) compute the 2D GWLD histogram for bovine iris image with these differential excitations and orientations of each pixel of each Gabor magnitude map, and then generate the more discriminative 1D GWLD histogram. The following subsections will describe these procedures in detail.

3.1.1. Gabor Magnitude Maps. Considering the advantages of the Gabor filter in object recognition $[4,24]$, we exploit the multiscale and multiorientation Gabor filters to de-composite the input bovine iris images for feature extraction. The Gabor representation of a bovine iris image is derived by convolving the iris image with the Gabor filters. Let $I(x, y)$ be the enhanced bovine iris image; its convolution with a Gabor filter is $\psi_{\mu, \nu}(z)$ defined as

$$
G_{\psi f}(x, y, \mu, \nu)=I(x, y) * \psi_{\mu, \nu}(z),
$$

where $*$ denotes the convolution operator. The Gabor filters we used are defined as follows:

$$
\psi_{\mu, \nu}(z)=\frac{\left\|k_{\mu, \nu}\right\|^{2}}{\sigma^{2}} e^{\left(-\left\|k_{\mu, \nu}\right\|^{2}\|z\|^{2} / 2 \sigma^{2}\right)}\left[e^{i k_{\mu, \nu} z}-e^{-\sigma^{2} / 2}\right],
$$




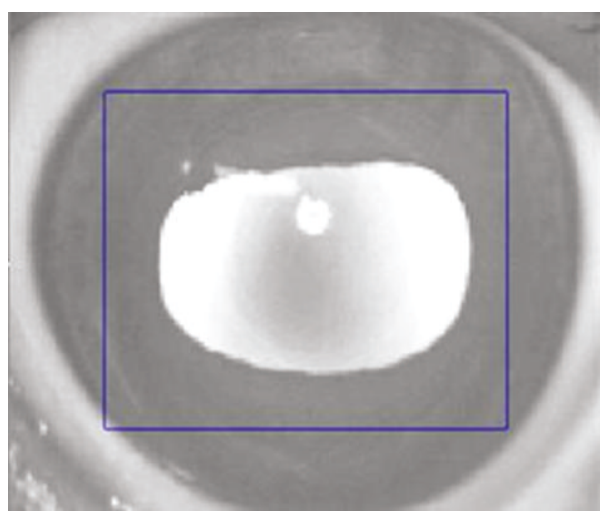

(a)

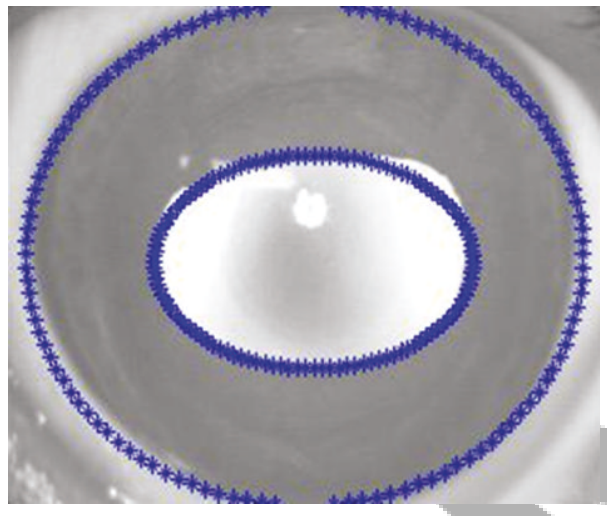

(c)

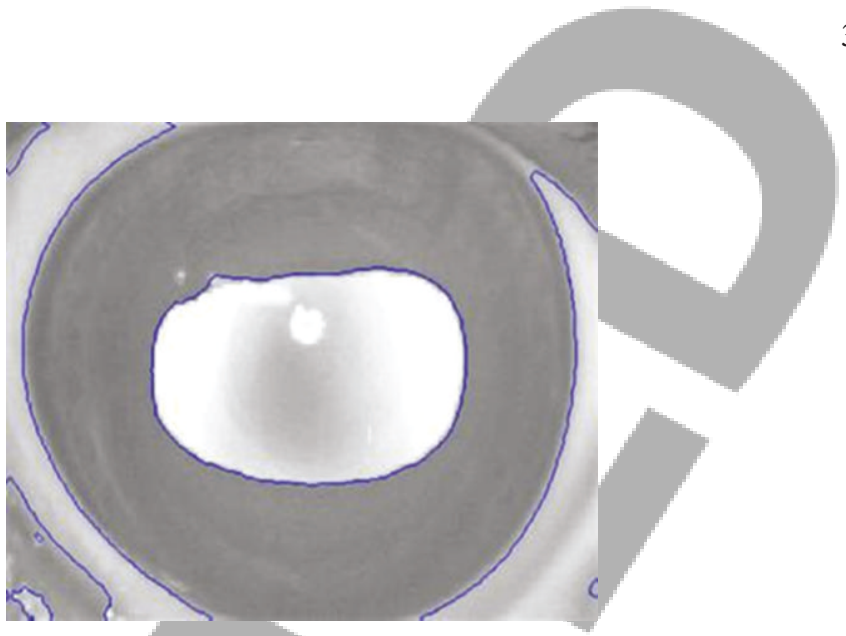

(b)

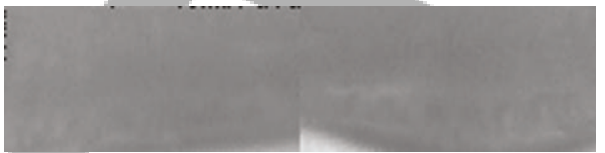

(d)

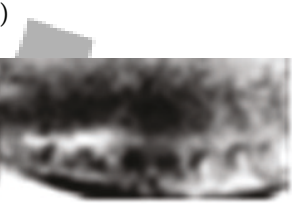

(e)

FIGURE 1: Preprocessing of the bovine iris image.

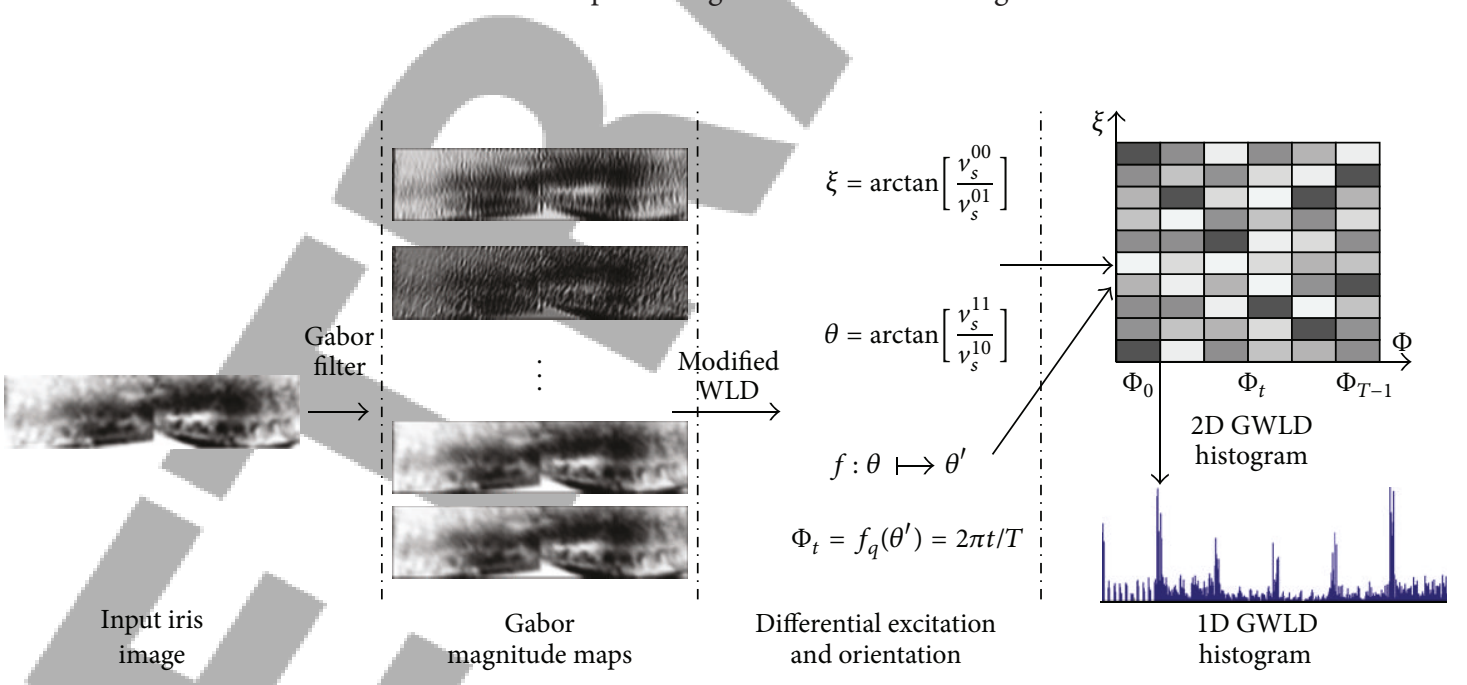

FIGURE 2: The framework of the proposed GWLD bovine iris representation approach.

where $\mu$ and $\nu$ denote the orientation and scale of the Gabor filters, respectively. $\|\cdot\|$ defines the norm operator, and the wave vector is defined as

$$
k_{\mu, \nu}=k_{\nu} e^{i \phi_{\mu}},
$$




\begin{tabular}{|l|l|l|}
\hline$x_{0}$ & $x_{1}$ & $x_{2}$ \\
\hline$x_{7}$ & $x_{c}$ & $x_{3}$ \\
\hline$x_{6}$ & $x_{5}$ & $x_{4}$ \\
\hline
\end{tabular}

Neighbor pixels

(a)

\begin{tabular}{|c|c|c|}
\hline 0 & 0 & 0 \\
\hline 0 & +1 & 0 \\
\hline 0 & 0 & 0 \\
\hline
\end{tabular}

$f_{01}$

(b)

\begin{tabular}{|c|c|c|}
\hline 0 & -1 & 0 \\
\hline 0 & 0 & 0 \\
\hline 0 & +1 & 0 \\
\hline
\end{tabular}

$f_{10}$

(c)

Figure 3: Neighbor pixels and filters $f_{01}, f_{10}$, and $f_{11}$.

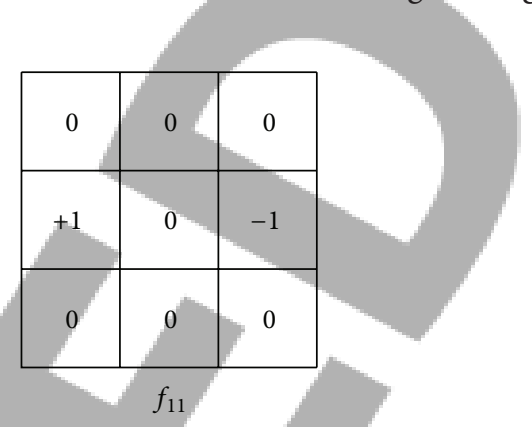

(d)

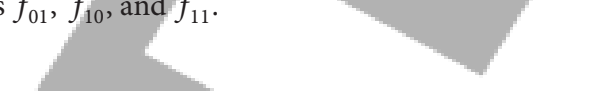

each input bovine iris image, while one magnitude value is computed at each pixel for each Gabor filter.

3.1.2. Differential Excitation and Orientation. Differential excitation and orientation are the two components to generate the WLD histogram of input image. Notice that there exist relatively flat areas in the input normalized bovine iris image. We apply the modified WLD operator [25] over each Gabor magnitude map to compute the differential excitation and orientation.

Differential excitation $\xi\left(x_{c}\right)$ represents the intensity difference between a current pixel $x_{c}$ and its neighbors. To compute $\xi\left(x_{c}\right)$, we first calculate the difference $v_{s}^{00}$ between a current pixel and its neighbors using the following filter $f_{00}$ :

$$
f_{00}=p *\left(x_{j}-x_{c}\right) \text {. }
$$

Here $x_{j}$ is the corresponding neighbor pixel of the Chebyshev distance

$$
x_{j}=\underset{x_{i}}{\arg \max }\left|x_{i}-x_{c}\right|, \quad i=0,1, \ldots, p-1,
$$

where $x_{i}$ denotes the $i$ th neighbor of $x_{c}$ and $p$ is the number of neighbors.

Then, we compute the ratio of the difference $v_{s}^{00}$ to the intensity of the current pixel $v_{s}^{01}$, which is the output of filter $f_{01}$ (as shown in Figure 3):

$$
G_{\text {ratio }}=\frac{v_{s}^{00}}{v_{s}^{01}}
$$

Finally, the differential excitation of the current pixel $x_{c}$ is computed as

$$
\xi\left(x_{c}\right)=\arctan \left[G_{\text {ratio }}\right]=\arctan \left[\frac{v_{s}^{00}}{v_{s}^{01}}\right] .
$$

Furthermore, we use $v_{s}^{10}$ and $v_{s}^{11}$, which are the outputs of filters $f_{s}^{10}$ and $f_{s}^{11}$ (as shown in Figure 3), to compute the orientation $\theta\left(x_{c}\right)$ for each pixel $x_{c}$ :

$$
\theta\left(x_{c}\right)=\arctan \left[\frac{v_{s}^{11}}{v_{s}^{10}}\right]
$$

where $v_{s}^{10}=x_{5}-x_{1}$ and $v_{s}^{11}=x_{7}-x_{3}$.
3.1.3. GWLD Histogram Representation. After calculating the differential excitation and orientation, we get the WLD features $\{\xi, \theta\}$ for each pixel of these 40 Gabor magnitude maps. Note that both ranges of differential excitation and orientation are $[-\pi / 2, \pi / 2]$. Same to WLD [22], the orientation of each pixel $\theta$ is mapping to the range $[0,2 \pi]$, and then it is quantized to $T$ dominant orientations $\Phi_{t}$. Then, we compute the 2D GWLD histogram with the differential excitation and the quantized orientation of each pixel of 40 Gabor magnitude maps. And in the 2D histogram, each column corresponds to a dominant orientation $\Phi_{t}$. After computing the $2 \mathrm{D}$ histogram, we further quantize the differential excitation $\xi$. The range of differential excitation $\xi$ is divided into $M$ subranges, and each subrange includes $S$ dominant differential excitations. Therefore, the 2D GWLD histogram can be encoded into a $1 \mathrm{D}$ histogram by regrouping the quantized differential excitation along with the dominant orientation [22], and the length of the 1D GWLD histogram representation is

$$
L=M \times S \times T \text {. }
$$

3.2. Iris Matching. Several similarity measurement approaches have been presented for histogram matching [26]. In this paper, we use the histogram intersection as the similarity measurement of two GWLD histogram representations. Suppose $\mathrm{GWLD}^{x}$ and $\mathrm{GWLD}^{y}$ are two GWLD histogram representations, and the histogram intersection is defined as

$$
\mathrm{Hi}\left(\mathrm{GWLD}^{x}, \mathrm{GWLD}^{y}\right)=\sum_{i=1}^{L} \min \left(\mathrm{GWLD}_{i}^{x}, \mathrm{GWLD}_{i}^{y}\right) \text {, }
$$

where $L$ is the length of the histogram.

The intuitive motivation for the histogram intersection measurement is the calculation of the common part of two histograms. It is clear that larger value of the histogram intersection means higher similarity of the two input bovine iris images.

\section{Experiments}

We apply the proposed descriptor to bovine iris to test its performance. We select 18 subjects, 90 original images from 

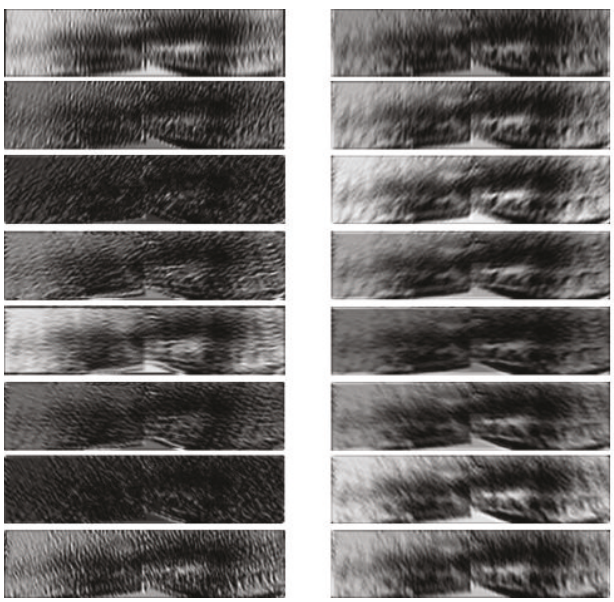

(a) Input bovine iris image
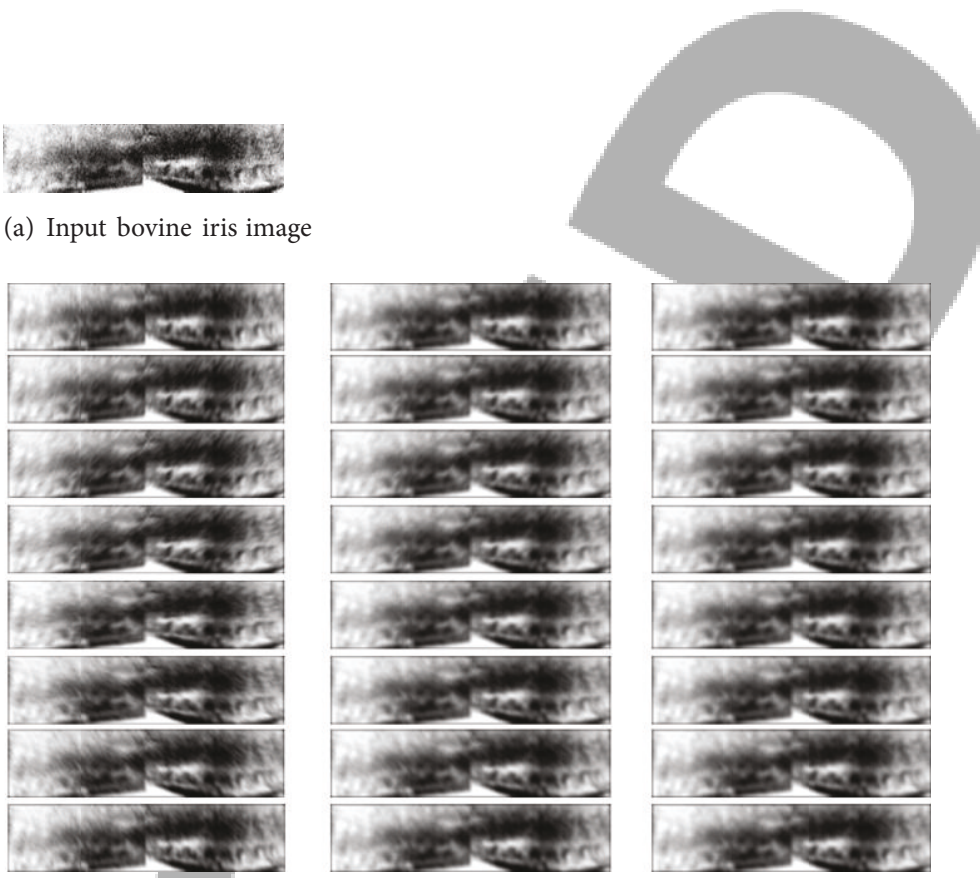

(b) Gabor magnitude maps with mask size $5 \times 5$
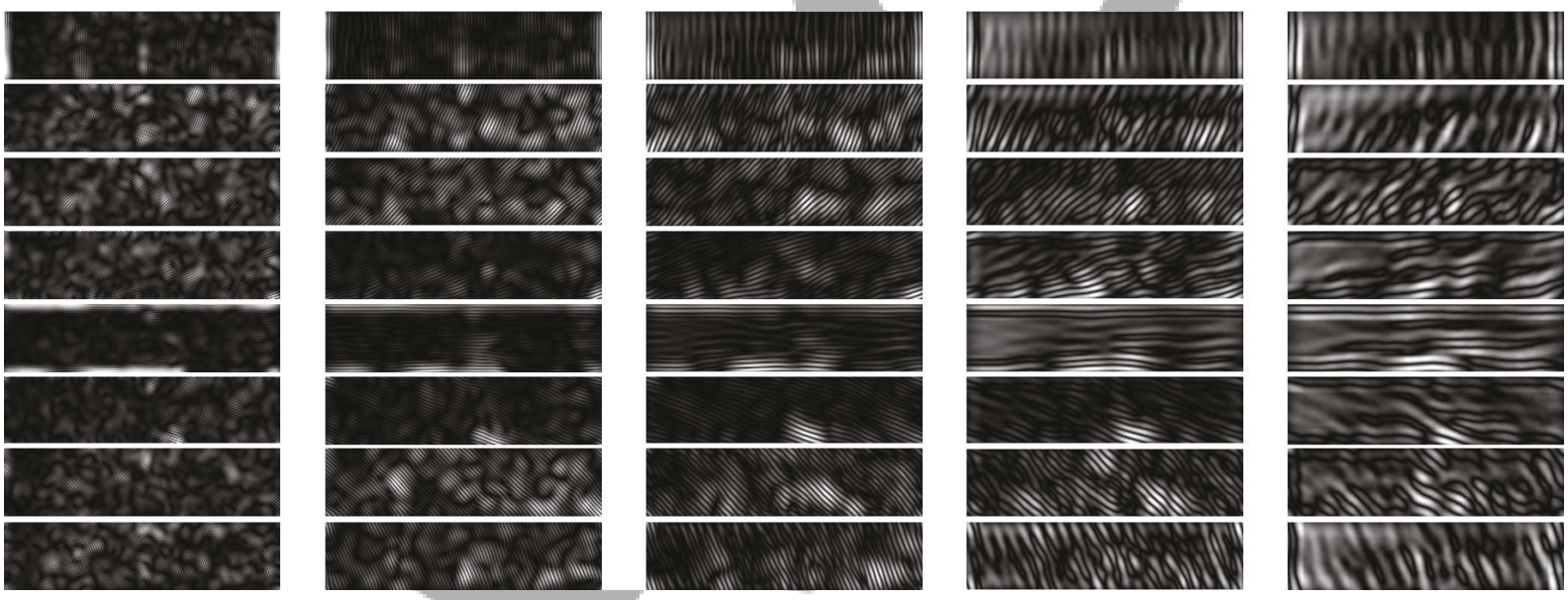

(c) Gabor magnitude maps with mask size $31 \times 31$

FIGURE 4: Different convolution masks of the Gabor filter.

the SEU bovine iris database. The size of the original iris images is $320 \times 240$, and the size of normalized iris image is $253 \times 61$. For each input bovine iris image, there are 40 Gabor magnitude maps after convolution. Therefore, $40 \times 253 \times 61$ pairs of quantized differential excitations and orientations are calculated to generate the $1 \mathrm{D}$ GWLD histogram. In addition, accurate recognition rate (ARR) is adopted to evaluate the performance of these experiments.

4.1. Parameters Assessment. According to these applications of WLD in iris recognition [25], face recognition [27], and pedestrian detection [28], the length of histogram representations affects the performance of the local descriptor. Furthermore, according to the aforementioned analysis, the first step of the proposed local descriptor GWLD is to generate these Gabor magnitude maps by convolving the input image with the Gabor filters. As shown in Figure 4, the results of different convolution mask sizes are quite different. Therefore, the mask size may affect the performance of GWLD.

Figure 5 illustrates the performance variation against the length of GWLD histogram and the convolution mask size. The ARR increases considerably with the rise in the length of GWLD histogram and reaches the maximum at $L=32000$. Nevertheless, it decreases when the length rises further. That is because the GWLD histogram gets much sparser while the number of the differential excitations and orientations is limited. In additional, large length $L$ increases the computation requirements of quantization of differential excitations and orientations.

Moreover, from Figure 5, one can see that GWLD with mask size $5 \times 5$ outperforms that with larger mask size. In other words, GWLD prefers these Gabor magnitude maps convolved with small mask size. Figure 4 illustrates that 


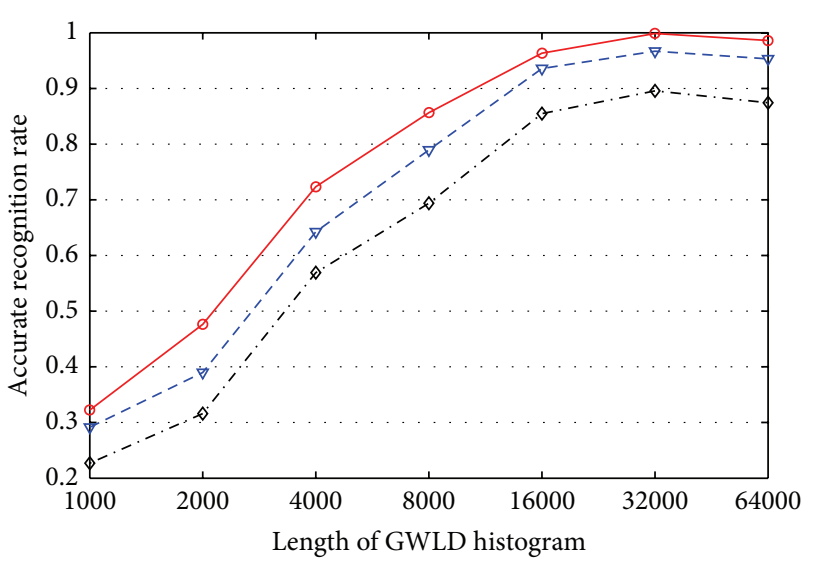

$\multimap-$ Mask size $5 \times 5$
$-\nabla-$ Mask size $13 \times 13$
$-\diamond-$ Mask size $31 \times 31$

FIgure 5: Performance of GWLD.

TABLE 1: Performance comparison among different feature descriptors.

\begin{tabular}{lc}
\hline Feature descriptor & ARR \\
\hline LBP [29] & $93.1 \%$ \\
Center-epsilon LBP [29] & $95.79 \%$ \\
WLD [25] & $96.20 \%$ \\
Modified WLD [25] & $98.73 \%$ \\
Gabor filter [23] & $98.30 \%$ \\
Proposed GWLD & $\mathbf{9 8 . 8 7} \%$ \\
\hline
\end{tabular}

Gabor magnitude maps with small convolution mask size $5 \times 5$ demonstrate more global features, while other maps with large mask size $(31 \times 31)$ show more local features.

4.2. Performance Comparison. Feature descriptor plays an important role in the iris recognition system. In this paper, we compare our proposed GWLD with LBP, center-epsilon LBP, WLD, modified WLD and Gabor filter on the same dataset to evaluate the performance of our proposed descriptor. As can be seen from Table 1, the LBP achieves the lowest accurate recognition rate, and the center-epsilon LBP gets a better performance (while epsilon equals 3 ), as it takes the central pixel information into consideration to generate the feature representation [29]. Meanwhile, the WLD operator shows a more discriminative ability than LBP, for its representation is generated from the intensity difference and the orientation information. And by applying a different computation scheme for the difference excitation, the modified WLD achieves a higher ARR [25]. Furthermore, as our proposed GWLD integrates the advantages of Gabor filter and modified WLD, it outperforms other feature descriptors with the highest ARR. In other words, our proposed feature descriptor is quite effective for bovine iris recognition.

\section{Conclusion and Future Work}

This paper has proposed a novel feature descriptor GWLD for bovine iris recognition. The GWLD constructs the histogram representation by computing the differential excitation and orientation over the Gabor magnitude maps of the input bovine iris image. Experiments on the SEU bovine iris database show that the proposed feature descriptor has encouraging performance, and the length of the GWLD histogram and the mask size of Gabor filters affect the recognition accuracy.

One problem of our approach might be that its computation cost is high, because the differential excitations and orientations are calculated over each Gabor magnitude map for each pixel. Hence, one of our future efforts is to reduce the computational cost with some feature selection strategies. Other problems might be its relatively high-dimensional histogram representation and the similarity measurement. The high dimensional histogram representation can be alleviated by applying some analysis approach (e.g., PCA) to the histogram, and its performance can be improved with more suitable similarity measurement (e.g., ADTW [30]). Furthermore, the applicability of the bovine iris recognition in food safety might be increased by other enhancements, for example, taking the motion images into consideration [31].

\section{Acknowledgments}

This work is partially supported by the National Key Technology R\&D Programs of China under Contract nos. 2013BAD19B05, 2006BAK02A16, and 2006BAK02A28 and the Scientific Research Foundation of Graduate School of Southeast University, China, under Contract no. YBJJ1034.

\section{References}

[1] F. Madec, R. Geers, P. Vesseur, N. Kjeldsen, and T. Blaha, “Traceability in the pig production chain," OIE Revue Scientifique et Technique, vol. 20, no. 2, pp. 523-537, 2001.

[2] W. Shirou, "Cost-effective product traceability system based on widely distributed database," Journal of Communications, vol. 2, no. 2, pp. 45-52, 2007.

[3] L. Zhao, S. Shengnan, and X. Wang, "Tracking and traceability system using livestock Iris code in meat supply chain," International Journal of Innovative Computing, Information and Control, vol. 7, no. 5A, pp. 2201-2212, 2011.

[4] J. G. Daugman, "High confidence visual recognition of persons by a test of statistical independence," IEEE Transactions on Pattern Analysis and Machine Intelligence, vol. 15, no. 11, pp. 1148-1161, 1993.

[5] A. Ross, "Iris recognition: the path forward," Computer, vol. 43, no. 2, pp. 30-35, 2010.

[6] P. Li, X. Liu, and N. Zhao, "Weighted co-occurrence phase histogram for iris recognition," Pattern Recognition Letters, vol. 33, no. 8, pp. 1000-1005, 2012.

[7] P. Yao, J. Li, X. Ye, Z. Zhuang, and B. Li, "Iris recognition algorithm using modified Log-Gabor filters," in Proceedings of the 18th International Conference on Pattern Recognition (ICPR '06), pp. 461-464, August 2006. 
[8] R. P. Wildes, "Iris recognition: an emerging biometrie technology," Proceedings of the IEEE, vol. 85, no. 9, pp. 1348-1363, 1997.

[9] L. Ma, T. Tan, Y. Wang, and D. Zhang, "Personal identification based on iris texture analysis," IEEE Transactions on Pattern Analysis and Machine Intelligence, vol. 25, no. 12, pp. 1519-1533, 2003.

[10] Z. Sun, T. Tan, and Y. Wang, "Robust encoding of local ordinal measures: a general framework of iris recognition," in Proceedings of the International Workshop on Biometric Authentication, vol. 3087, pp. 270-282, 2004.

[11] D. M. Monro, S. Rakshit, and D. Zhang, "DCT-bsed iris recognition," IEEE Transactions on Pattern Analysis and Machine Intelligence, vol. 29, no. 4, pp. 586-595, 2007.

[12] K. Miyazawa, K. Ito, T. Aoki, K. Kobayashi, and H. Nakajima, "An effective approach for Iris recognition using phase-based image matching," IEEE Transactions on Pattern Analysis and Machine Intelligence, vol. 30, no. 10, pp. 1741-1756, 2008.

[13] T. Ojala, M. Pietikäinen, and T. Mäenpää, "Multiresolution gray-scale and rotation invariant texture classification with local binary patterns," IEEE Transactions on Pattern Analysis and Machine Intelligence, vol. 24, no. 7, pp. 971-987, 2002.

[14] D. G. Lowe, "Distinctive image features from scale-invariant keypoints," International Journal of Computer Vision, vol. 60, no. 2, pp. 91-110, 2004.

[15] E. Tola, V. Lepetit, and P. Fua, "DAISY: an efficient dense descriptor applied to wide-baseline stereo," IEEE Transactions on Pattern Analysis and Machine Intelligence, vol. 32, no. 5, pp. 815-830, 2010.

[16] Z. Sun, T. Tan, and X. Qiu, "Graph matching iris image blocks with local binary pattern," in Proceedings of the International Conference on Biometrics, vol. 3832, pp. 366-372, 2006.

[17] C. Belcher and Y. Du, "Region-based SIFT approach to iris recognition," Optics and Lasers in Engineering, vol. 47, no. 1, pp. 139-147, 2009.

[18] M. Zhang, Z. Sun, and T. Tan, "Deformable DAISY matcher for robust iris recognition," in Proceedings of the IEEE Conference on Image Processing, pp. 3250-3253, 2011.

[19] W. Zhang, S. Shan, W. Gao, X. Chen, and H. Zhang, "Local Gabor Binary Pattern Histogram Sequence (LGBPHS): a novel non-statistical model for face representation and recognition," in Proceedings of the 10th IEEE International Conference on Computer Vision (ICCV'05), pp. 786-791, October 2005.

[20] K. Takahashi, Y. Kuriya, and T. Morie, "Bicycle detection using pedaling movement by spatiotemporal Gabor filtering," International Journal of Innovative Computing, Information and Control, vol. 8, no. 6, pp. 4059-4070, 2012.

[21] N. Dalal, B. Triggs, and C. Schmid, "Human detection using oriented histograms of flow and appearance," in Proceedings of the European Conference on Computer Vision, vol. 3952, pp. 428441, 2006.

[22] J. Chen, S. Shan, C. He et al., "WLD: a robust local image descriptor," IEEE Transactions on Pattern Analysis and Machine Intelligence, vol. 32, no. 9, pp. 1705-1720, 2010.

[23] S. Sun and L. Zhao, "Bovine iris segmentation using regionbased active contour model," International Journal of Innovative Computing, Information and Control, vol. 8, no. 9, pp. 64616471, 2012.

[24] C. Liu and H. Wechsler, "Gabor feature based classification using the enhanced Fisher linear discriminant model for face recognition," IEEE Transactions on Image Processing, vol. 11, no. 4, pp. 467-476, 2002.
[25] S. Sun and L. Zhao, "Efficient modified Weber local descriptor approach to bovine iris recognition," ICIC Express Letters Part B, vol. 3, no. 5, pp. 1311-1317, 2012.

[26] Y. Rubner, J. Puzicha, C. Tomasi, and J. M. Buhmann, "Empirical evaluation of dissimilarity measures for color and texture," Computer Vision and Image Understanding, vol. 84, no. 1, pp. 25-43, 2001.

[27] D. Gong, S. Li, and Y. Xiang, "Face recognition using the Weber local descriptor," in Proceedings of the Asian Conference on Pattern Recognition, pp. 589-592, 2011.

[28] G. Lian, J. Lai, and Y. Yuan, "Fast pedestrian detection using a modified WLD detector in salient region," in Proceedings of the International Conference on System Science and Engineering, pp. 564-569, 2001.

[29] Y. Song, S. Sun, and L. Zhao, "Learning center-epsilon local binary pattern for bovine iris recognition," ICIC Express Letters, vol. 7, no. 4, pp. 1209-1214, 2013.

[30] S. Adwan and H. Abof, "Modified integral projection method for eye detection using dynamic time warping," International Journal of Innovative Computing, Information and Control, vol. 8, no. 1A, pp. 187-200, 2012.

[31] A. Eftakhar, J. Tan, H. Kim, and S. Ishikawa, "An effective directional motion database organization for human motion recognition," International Journal of Innovative Computing, Information and Control, vol. 8, no. 2, pp. 1359-1370, 2012.

\footnotetext{
-
}

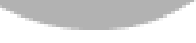

